\title{
La internacionalización de la Educación Superior: experiencias de los servicios académicos de la Facultad Turismo en Angola. Logros y retos.
}

The internationalization of Higher Education: experiences of the academic services of the Faculty of Tourism in Angola. Achievements and challenges.

\author{
MSc. Yanela González González ${ }^{1}$
}

\section{Resumen.}

Luego de varias reuniones de trabajo y la nueva creación del ministerio de Enseñanza Superior en el 2012, comenzó a coordinarse un plan de acciones que incluían 12 carreras con el plan cubano, para la impartición de clases en Angola, por profesores cubanos. Una de esas carreras fue la de la Licenciatura de turismo, dando respuesta a una necesidad de formación de recursos humanos en esta área. Se contó con la experticia de profesores en el sector, quienes dedicaron su obra para elaborar planes de estudios, organización escolar, adaptaciones al territorio, representando a la dirección del país en este empeño.

Con este trabajo proponemos un acercamiento general a los antecedentes de esta colaboración, acciones gubernamentales y resultados potenciales desde la investigación científica de manera general y desde la internacionalización de la educación superior.

Palabras claves: solidaridad, turismo, internacionalización, educación superior, colaboración, investigación.

\begin{abstract}
.
After several work meetings and the new election of the minister of higher education in 2012, a plan of actions that included 12 careers with the Cuban plan began to be coordinated to teach in Angola by Cuban professors. One of those careers was that of the tourism degree, responding to a need for training of human resources in this area. it was counted on the expertise of professors in the sector, who dedicated their work to elaborate plans of studies, scholastic organization, adaptations to the territory, representing to the direction of the country in this endeavor. With this work we propose a general approach to the background of this collaboration, government actions and potential results from scientific research in general.
\end{abstract}

\footnotetext{
${ }^{1}$ Universidad de La Habana, Facultad de Turismo. La Habana, Cuba. ygg2180@gmail.com yanela.gonzalez@ftur.uh.cu
} 
Keywords: solidarity, tourism, international, high education, colaboration, resource.

\section{Introducción}

Para la celebración de las elecciones en Angola en agosto de 2012, se crearon las bases para la apertura del Ministerio de Enseñanza Superior, separándose de la labor de Ciencia y Tecnología. Para febrero de 2013, ya participaba en el Congreso "Pedagogía 2013", el Sr. Adao do Nascimento, recién electo Ministro, acompañado de una amplia delegación. Como resultado de esta visita de intercambios y análisis, se firmaron las Memorias de trabajo entre ambos Ministerios, que devino en la ejecución de un amplio y ambicioso Plan de Acciones que contempla los servicios Académicos, la movilidad de estudiantes y profesores, asesorías en la creación de carreras, Centros de Investigaciones y la continuación del envío de profesores cubanos para la impartición de la docencia.

Para entender la tarea que asumieron los profesores cubanos en la creación de estas nuevas carreras, se tuvo en cuenta que la República de Angola está situada en África Austral,ocupa un área de $1.246 .700 \mathrm{~km}^{2}$ cuya población estimada es de 14.602 .002 habitantes, en 2002.

Es un país plurilinguístico, donde o Português es la lengua oficial de comunicación entre los angolanos. .

La República de Angola, un vasto país, poblado, estuvo colonizada desde 1482 por los portugueses. El 11 de noviembre de 1975, obtuvieron la independencia.

Haciendo un poco de historia, Portugal no logró dominar todo el territorio desde los primeros contactos, hicieron falta siglos para que lograra imponer su dominio político y económico a todo el pueblo. En este país siempre hubo intereses de otras potencias occidentales, asociados del poder colonial, como Gran Bretaña, con importantes inversiones, los EUA, con crecientes intereses en la economía y ansiando dominar la posición estratégica de Angola. Por lo tanto fue un reto que asumieron a pesar de la lejanía, las diferencias culturales, la implementación de planes de estudios de carreras como la de Licenciatura en Turismo.

Para adentrarnos en el tema de la Internacionalización de la Educación Superior en este país debemos partir de una idea: no encontramos en África un único país que no mantenga relaciones preferenciales con su antigua metrópoli, incluso por la absorción de los valores culturales inevitable en un régimen de tipo colonial. Y más, las formas de explotación no terminaron, como consecuencia, no terminaron tampoco las formas de discriminación racial, más o menos acentuadas. Es que, en estos casos la liberación todavía no es completa. Al terminar la guerra, muchos jóvenes fueron repatriados, la mayoría con nivel superior y para lograr la especialización, maestrías y doctorados, quienes les ofrecían mejores eran universidades portuguesas, evidenciándose aún los intereses de la ex colonia.

El líder angolano A. Neto expresó en una conferencia: ... “ lucha del pueblo por el poder político, por la independencia económica, por el restablecimiento de la vida cultural, por la desalineación, por las relaciones con todos los pueblos, en una base de igualdad y de 
fraternidad, tales son los objetivos de nuestra lucha. Y Cuba desde el principio cumplió con esta base fundamental para lograr la independencia nacional, pues desde el comienzo apoyó para alcanzar la liberación de este país. Asimismo constituyó la primera acción para la enseñanza de las futuras generaciones en tiempo de guerra. Ambos gobiernos creyeron necesario preparar ideológicamente desde el punto de vista de la defensa de la soberanía nacional y en post de su desarrollo.

Es por ello que cuando hablamos de la Internacionalización de la Educación Superior para abordarlo desde la arista de la cooperación internacional entre Angola y Cuba, partimos de la siguiente idea: es el proceso de integrar una dimensión internacional, intercultural y global en la docencia, la investigación y el servicio y la extensión de la Educación Superior. Con el objetivo de mejorar su calidad y elevar el nivel académico de los programas de Educación superior y de los profesores y estudiantes que participan en ellos.

\section{Desarrollo.}

Desde el siglo pasado la internacionalización de la Educación Superior se potenció y se desarrolló a través de una creciente cooperación internacional favorecida desde los años 90 del siglo XX por el incremento de la internet, de las tecnologías de información y comunicación, al igual que la emergencia de espacios virtuales. En la última década la internacionalización de la Educación Superior ha tenido una enorme expansión. Los intercambios de estudiantes y profesores en el ámbito de la formación, la docencia y la investigación han implicado una renovación, ampliación y consolidación de la cooperación internacional entre las universidades.

Ya lo auguraba, António Agostinho Neto líder revolucionario de la República de Angola, fundador del MPLA, que condujo a su pueblo a la independencia y fue el primer presidente de este país. En uno de los discursos pronunciados por él en universidades africanas dijo: los lazos históricos que vinculan nuestros pueblos a los otros pueblos del mundo se van a estrechar cada vez más, puesto que no puede haber otra tendencia sobre la tierra. El aislamiento es imposible, y es contrario a la idea del progreso técnico, cultural y político. ${ }^{2}$

Hay que reflexionar sobre los valores que deben fundar la misión universitaria para no sucumbir a la mercantilización de la Educación Superior. La globalización está cuestionando el principal valor de la Universidad: su servicio al bien común y al conjunto de la sociedad.

Con respecto al servilismo mercantil que podía sumir a los países africanos dijo: ... el problema que se nos plantea en este momento es cómo transformar las relaciones injustas, generalmente de subordinación política y económica, con otros países y pueblos del mundo, sin que esa transformación se haga en perjuicio del progreso social que necesariamente debe estar inoculado en la acción por la libertad, y sin que el comportamiento del hombre sea el de quien sale de una forma de discriminación para caer

${ }^{2}$ Conferencia en la Universidad de Dar es Salaam, Tanzania, el 7 de febrero de 1974, pág 7-8. 
en otra forma tan negativa como la primera, como pura inversión de los factores intervinientes. $^{3}$

Es importante diferenciar entre internacionalización y trasnacionalización de la Educación Superior. La interacción está fundada en el valor del conocimiento y de la formación y se refiere a las diversas formas de cooperación entre instituciones de Educación Superior (IES), investigadores, profesores, estudiantes. La trasnacionalización, en cambio, tiene como principios esenciales la competitividad y el ánimo de lucro.

Si bien las tecnologías de la información desempeñan un importante papel en la constitución de redes de universidades a escala mundial y esto ha hecho posible "internacionalizar los programas de estudio, también es cierto que la interacción tiene aspectos preocupantes, pues cuando los estudiantes, una vez obtenido el diploma, no regresan a su país, esto contribuye a la "fuga de cerebros" y a una transferencia de reservas tecnológicas. ${ }^{4}$

Apertura de la carrera de licenciatura en turismo. Apuntes sobre cooperación Internacional.

Sobre estas bases se priorizó el envío de profesores para la apertura de la carrera de Turismo en la República de Angola. A través de ANTEX se envió un plan de estudio que tuviera las características esenciales de este sector en Angola, pues se previó una colaboración en la formación para posibles intercambios, colaboración y el incremento de profesores cubanos contratados con esta finalidad.

Todos los elementos antes tratados que reflejan las últimas tendencias, han estado presente en las intenciones de ambos gobiernos para formar recursos humanos más capaces, integrales, revolucionarios y comprometidos con la educación universitaria de las futuras generaciones.

Partimos del criterio que la cooperación internacional no es más que: en su acepción más general, a los esfuerzos de colaboración entre 2 o más países para abordar una problemática que afecte a uno de ellos o a varios, o para afrontar temas de interés común. En una definición más colindante, el término cooperación internacional, se refiere a lo que se conoce como Ayuda Oficial al Desarrollo-AOD- ${ }^{5}$. De acuerdo con el Committe for Development (OECD), la AOD debe cumplir 3 requisitos fundamentales:

a) Ser llevada a cabo por los gobiernos en carácter oficial.

b) Tener como principal objetivo la promoción del desarrollo económico, y el bienestar de los países en desarrollo.

c) Ser de carácter concesional y contener un elemento de donación de al menos el $25 \%$.

\footnotetext{
${ }^{3}$ Idem.

${ }^{4}$ I.CIC-F10C: Globalización y enseñanza superior católica, p16. Documento de Trabajo. 2004

${ }^{5}$ L/T: La Internacionalización de la Educación Superior a nivel mundial y regional. Principales tendencias y desafíos. Humberto Grimaldo Durán, Francisco López Segrera, págs.:200-246
} 
Asumimos las ideas expresadas en documentos publicados por la Agencia Presidencial para la Acción Social de Colombia, que presenta la siguiente definición: "la Cooperación Internacional es la ayuda que se entrega para apoyar el desarrollo económico y social de países en desarrollo mediante la transferencia de tecnologías, conocimientos, habilidades o experiencias por parte de países u organizaciones multilaterales."

En el mismo documento se define la Cooperación Técnica como ..." la ayuda que se entrega mediante la transferencia de técnicas, tecnologías, conocimientos, habilidades o experiencias por parte de países, con el fin de apoyar el desarrollo socioeconómico de los países con menor nivel de desarrollo tecnológico, la formación de recursos humanos y mejorar la capacidad de las instituciones.

Cooperación en la carrera de licenciatura en turismo.

En nuestro caso partimos del criterio de una cooperación solidaria con la República de Angola, que se caracteriza por la no injerencia en asuntos internos, la ausencia de condicionantes explícitas, la horizontalidad (igualdad) entre los países socios, la promoción de la autosuficiencia, la preferencia por la utilización de recursos locales que generen mayores incentivos para la apropiación, la adaptación a las prioridades de los países socios y un menor costo relativo sin sacrificar eficiencia.

En el año 2001, el Ministerio de Educación Superior de Cuba, a través de Convenio firmado con la Corporación ANTEX S.A., Empresa de Servicios Técnicos y Profesionales en el Exterior, reanuda la colaboración académica, con la presencia de 5 profesores cubanos, en la Universidad Agosthino Neto de Angola.

Nuestras relaciones de cooperación académica, científico- técnicas y de formación de cuadros han estado amparadas en convenios intergubernamentales y en la XII Sesión entre ambos países que se celebró en la Habana, en el mes de febrero del 2006.

Durante la visita del Presidente José Eduardo Dos Santos a Cuba, en septiembre del 2007, quien viajó acompañado del Sr. Adao Gaspar Ferreira Do Nascimento, Secretario de Estado para la Educación Superior y otros funcionarios angolanos, se suscribieron varios Acuerdos de Cooperación y Memorandos de Entendimiento tales como:

$>$ Acuerdo de Cooperación entre el Gobierno de la República de Cuba y el Gobierno de la República de Angola en la esfera de la Enseñanza Superior y la Formación de cuadros (Firmado por el Ministro de Educación Superior de Cuba y el Secretario de Estado para la Enseñanza Superior de Angola).

$>$ Memorando de Entendimiento entre el Ministerio de Educación Superior de la República de Cuba y la Secretaría de Estado para la Enseñanza Superior de la República de Angola para la implementación del Acuerdo de Cooperación en la esfera de la Enseñanza Superior y la formación de cuadros (Firmado por el Ministro de Educación Superior de Cuba y el Secretario de Estado para la Educación Superior de Angola)

A partir de los Acuerdos firmados, se han desarrollado un conjunto de acciones que han contribuido, al incremento de la cantidad de profesores del Ministerio de Educación 
Superior de Cuba en Angola, para apoyar la docencia tanto en pregrado como en posgrado.

A partir del 2014, un grupo de profesores de la carrera de turismo, a solicitud del Ministerio de Educación Superior, realizaron un peritaje de 3 meses para identificar las verdaderas necesidades de colaboración entre ambos países, para la inserción de esta carrera en el sistema de la enseñanza superior en Angola.

Esto trajo consigo el inicio del curso escolar en septiembre de ese mismo año y con la matrícula aproximadamente de 200 estudiantes en las provincias de: Malange, Kuando kubango y Luanda. Para su inserción trabajaron sobre la base del Sistema de Enseñanza de la Educación.

La Ley Constitucional Angolana consagra a la Educación como un derecho de todos los ciudadanos, independientemente del sexo, raza, etnia e creencia religiosa.

En 1977, dos años después de la independencia nacional, fue aprobado un nuevo Sistema Nacional de Educación y Enseñanza que se basa en los siguientes Principios:

Principios de la Educación Angolana.

- Igualdad de oportunidades en el acesso y continuación de estudios;

- Gratuidades en todos los niveles de la Enseñanza.

- Perfeccionamiento constante del personal docente.

Sobre la Enseñanza Superior en la República de Angola, se emitió la ley 13/01 del 31 de diciembre de 2001, que garantizó la creación de un Subsistema de Educación Superior, la clasificación de las instituciones Universitarias (Universidades, Academias, Institutos Superiores (Técnicas y Politécnicas) y Escuelas Superiores (Técnicas y Politécnicas)), las estructuras Universitarias: Faculdades; Institutos Superiores; Escuelas Superiores; Centros de Estudios y de Investigación Científica, la Organización de Instituciones de la enseñanza Superior (Instituciones Públicas, Privadas y Público-Privadas.)

Los profesores tenían en cuenta para la confección del programa datos que ofrecemos a continuación para la pertinencia de la propuesta que se le presentó al Ministerio de Enseñanza Superior.

La población universitaria con cierre 2011, ascendía a más de 116.805, para un total de estudiantes en Instituciones Privadas de 55180 y en las públicas de 61625 aproximadamente.

Los docentes cubanos se encuentran aún distribuidos por estas 8 regiones académicas, definidas por la Secretaría de Estado a comienzos de la cooperación con la isla.

Una de las primicias que se tuvo en cuenta fue la colaboración en la formación de recursos humanos en Cuba desde el año 1961 hasta la actualidad, donde se han graduado en Cuba más de 9000 becarios de este país en las carreras prioritarias vinculadas a las Ciencias técnicas, económicas y pedagógicas. 
En el transcurso de estos años, el Ministerio de Educación Superior de Cuba ha contado con la presencia de más de 400 profesores de sus universidades en Angola.

Actualmente en la Facultad de Turismo y en otras provincias, hay más de 50 estudiantes en todo el país con el objetivo de formar futuros licenciados del sector en este país. Según criterios de profesores que prestaron servicios allá, no existe una planificación para la ubicación de los estudiantes en ninguna de las dos modalidades: los formados en Cuba y los formados por profesores cubanos en Angola. Ciertamente es una de las mayores debilidades que posee el sector del turismo, pues la mayoría de las instalaciones son propiedades privadas y convenidas con administraciones multinacionales, que no responden al gobierno directamente. Por lo que uno de los principales retos que enfrentarán los estudiantes es, la búsqueda de opciones laborales en ese sector.

Desde la facultad proponemos que se trabaje en algunas de estas áreas:

Principales líneas de la cooperación.

* Bolsa de Estudio de curso de graduación y posgraduación;

* Asesoría en la implementación y el desarrollo de cursos en áreas del sector turístico en 5 regiones académicas;

* Prestación de servicios de docentes cubanos en diferentes universidades angolanas; (desde los posgrados y asesorías...)

* Intercambio de información técnico-científica;

* Participación en eventos Internacionales;

Desafíos en esta cooperación Cuba- Angola.

Mucha ha sido la experiencia por los profesores cubanos en universidades angolanas, que han enfrentado el problema del idioma y se han preparado en educar e instruir a los estudiantes angolanos y docentes también, sobre todo en la identificación de herramientas para la investigación científica.

Podemos afirmar que los desafíos están en:

- La elaboración de proyectos de investigación conjunta;

- El incremento del número de docentes Cubanos en IES Angolanas;

- El incremento del número de Bolsas de Estudio de Posgrados;

- En implementar un Sistema de Superación Pedagógica de docentes;

- La concepción de proyectos conjuntos para coordinar cursos de posgraduación.

¿Qué se ha logrado hasta el momento con la cooperación internacional, desde los servicios académicos brindado por profesores cubanos en nuestras universidades cubanas $\mathrm{y}$ angolanas en la carrera de turismo?

- Mayor calidad en la formación pedagógica.

- Mayor pertinencia en las universidades angolanas, logrando un perfil más amplio en la formación de recursos humanos, más competentes y comprometidos con potenciar el sector del turismo para el futuro desarrollo de los angolanos. 
- Implantar mecanismos de seguimiento y evaluación a los programas educativos que promuevan el mejoramiento continuo y el interés de empresarios angolanos por ampliar las posibilidades de administrar los hoteles por nativos, donde se defienda y promueva lo autóctono.

- Incorporar a estudiantes, docentes e investigadores en dinámicas mundiales de $\mathrm{ES}$, que les permitan intercambiar conocimientos y experiencias y desarrollar iniciativas conjuntas que favorezcan el desarrollo nacional e internacional.

- Promover, mediante acciones determinadas y estratégicas, el aprendizaje y perfeccionamiento del idioma portugués y español, tanto en estudiantes de pregrado y posgrado como en los docentes. (Aunque aún es insuficiente la preparación de los docentes cubanos en el idioma portugués)

- Fortalecer el Sistema Nacional de Becarios Extranjeros ya sea por la vía autofinanciada (el gobierno asume el gasto de los estudiantes de más bajos ingresos) o no, que favorece a estudiantes con perfil socioeconómico bajo, pertenecientes a los niveles más humildes de la población angolana.

Ciencia, Tecnología e Innovación.

- En una sociedad del conocimiento cada vez más internacionalizada, el propósito de ambos países, es aumentar la investigación científica y el desarrollo tecnológico en las siguientes áreas estratégicas que impulsan el desarrollo económico y social del país.

a) Ambiente y biodiversidad.

b) Salud y vida.

c) Turismo sostenible.

d) Desarrollo organizacional, económico-industrial.

e) Recursos minerales y materiales.

f) Tecnologías de la Información y las Comunicaciones en el sector del turismo.

g) Construcción de ciudadanía e inclusión social.

h) Hábitat, ciudad y territorio.

i) Creación de Centro de convenciones y eventos.

j) Centros de documentación turística.

Ambos países parten del nuevo paradigma de la Responsabilidad Social, la universidad debe superar el enfoque de la "proyección social y extensión universitaria" como apéndices bien intencionados a su función central de formación estudiantil y producción de conocimientos, para poder asumir la verdadera exigencia de la Responsabilidad Social Universitaria.

La cooperación entre ambos países ha posibilitado la presencia de los docentes angolanos en foros de la Educación Superior, en la realización en las propias Universidades de Jornadas Científicas, Conferencias y en los Congresos de Universidad, que se realizan en Cuba, Angola constituye el primer país de África, que más participación tuvo y en la exposición de y defensa de ponencias y conferencias magistrales, enfatizando que la 
cooperación ha tenido sus frutos y que la confianza en el Sistema de la Educación Superior en Cuba aumenta cada día.

\section{Conclusiones}

$>$ A pesar del desarrollo de la cooperación internacional y al consenso que existe a escala mundial acerca de la importancia de la Educación Superior, las desigualdades se agudizan, la República de Angola y de Cuba, se esfuerzan porque estás desigualdades desaparezcan, y se evidencie que la cooperación solidaria a través de los Servicios Académicos internacionales es posible y la carrera de turismo en Angola, es una muestra de ello que ya en este curso arribará a su primera graduación.

$>$ Por otra parte se incrementa cada vez más la fuga de cerebros de los países en vías de desarrollo hacia los países desarrollados y de conjunto ambos países, priorizan que los estudiantes graduados angolanos, regresen a ponerse al servicio de su país, aunque no existan las condiciones creadas para este personal altamente calificado para su ubicación.

$>$ Ambos Ministerios se implican en su entorno, priorizando temas de investigación relevantes para la sociedad donde reside, eliminando de esta manera convertirse en una suministradora de élites que una vez formadas, emigrarán hacia los países desarrollados.

$>$ Aún se debe trabajar en que los profesores cubanos que imparten clases en las universidades angolanas de la carrera de turismo y otras, posean el dominio del idioma, evitando el uso del portuñol, no válido desde la estrategia de internacionalización de los sistemas de Educación Superior.

\section{Referencias Bibliográficas}

Conferencia Mundial sobre la Educación Superior-2009: La nueva dinámica de la Educación Superior y la Investigación para el cambio social y el desarrollo. Internacionalización, Regionalización y Mundialización.

Francois Vallaeys ¿Qué es la Responsabilidad Social Universitaria? http://palestra.pucp.edu.pe/index.php?id=111

L/T: La Internacionalización de la Educación Superior a nivel mundial y regional. Principales tendencias y desafíos. Humberto Grimaldo Durán, Francisco López Segrero. Edit: Planeta, Colombia S.A. Negocios Corporativos, 1ra Edic. 2012.

Real Thivierge, Guy. Secretario General de la Federación Internacional de Universidades Católicas (FIUC)

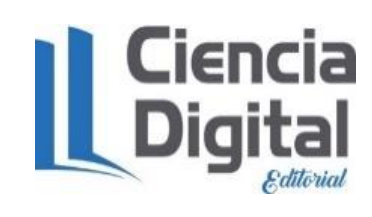




\section{Para citar el artículo indexado.}

González Y. (2019). La internacionalización de la Educación Superior: experiencias de los servicios académicos de la Facultad Turismo en Angola. Logros y retos. Revista electrónica Explorador Digital 3(1), 39-48. Recuperado desde:

http://cienciadigital.org/revistacienciadigital2/index.php/exploradordigital/article/view/345/7 $\underline{57}$

\section{Ciencia \\ Digital \\ Editorial}

El artículo que se publica es de exclusiva responsabilidad de los autores y no necesariamente reflejan el pensamiento de la Revista Explorador Digital.

El articulo queda en propiedad de la revista y, por tanto, su publicación parcial y/o total en otro medio tiene que ser autorizado por el director o editor de la Revista Explorador Digital.
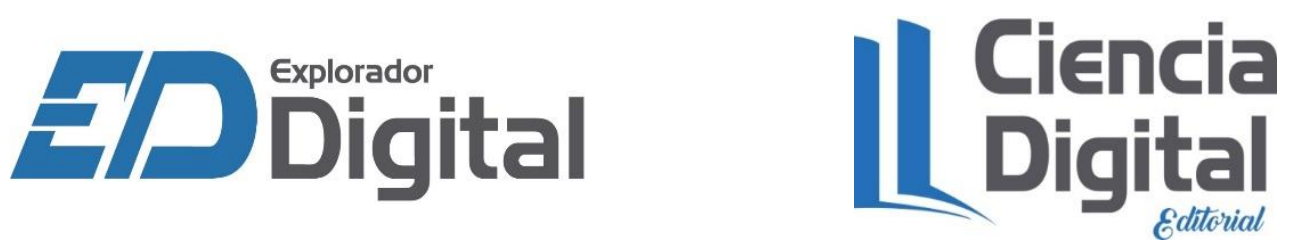